\title{
Evaluación de la microfiltración en restauraciones con resina Clase I.
}

\section{Evaluation of microfiltration in restorations with resin Class I.}

\author{
Laura María Cáceres Díaz ${ }^{1}$, Heriberto Núñez ${ }^{1}$, Mirtha Perdomo ${ }^{2}$
}

\section{RESUMEN}

Objetivo: Comparar la profundidad de microfiltración en restauraciones con resina en cavidades Clase I, variando el método de restauración y el sistema adhesivo. Material y Métodos: se realizó un estudio experimental ex vivo, en el que se incluyeron 128 molares sin alteraciones morfológicas en la estructura coronaria, en los cuales se tallaron cavidades en la cara oclusal en forma estandarizada $(4 \times 4 \times 3 \mathrm{~mm})$ y posteriormente distribuidos en forma aleatoria en dos grupos según el sistema adhesivo aplicado (Grupo I, grabado ácido previo al adhesivo; Grupo II, adhesivo autograbado), subdividiendose a su vez cada grupo en dos subgrupos según el método de restauración, A: resina compuesta; B: base de resina fluida, restauración con resina compuesta y sellador superficial. Todos los cuerpos de prueba fueron sellados en el ápice y en toda la superficie externa con dos capas de esmalte de uñas hasta $1 \mathrm{~mm}$ de la interfase diente-restauración, termociclados entre $5{ }^{\circ} \mathrm{C}$ y $55^{\circ} \mathrm{C}$, sumergidos en azul de metileno al $2 \%$ durante 24 horas y cortados transversalmente en sentido vestíbulo palatino/lingual para realizar la medición en milímetros. Resultados: Los datos fueron analizados estadísticamente con IBM SPSS stadicts versión 2.0, aplicando ANOVA de doble clasificación; se observó diferencia estadísticamente significativa $(\mathrm{p}<0,05)$ entre los subgrupos según el método de restauración. Conclusiones: la utilización de base y sellador superficial en combinación con la restauración de resina compuesta disminuyeron la microfiltración aplicando tanto el sistema adhesivo con grabado ácido previo como el sistema adhesivo autograbado, mientras que la mayor microfiltración se obtuvo con resina compuesta (sin base ni sellador superficial) y la utilización del sistema adhesivo autograbado.

PALABRAS CLAVE: microfiltración, resinas compuestas, adhesivos.

\section{ABSTRACT}

Objective: To compare the depth of microfiltration in resin restorations in Class I cavities, varying the restoration method and the adhesive system. Material and Methods: an ex vivo experimental study was carried out, in which 128 molars without morphological alterations in the coronary structure were included, in which cavities were carved in the occlusal face in a standardized way $(4 \times 4 \times 3 \mathrm{~mm})$ and later randomly distributed in two groups according to the adhesive system applied (Group I, acid etching prior to the adhesive; Group II, self-etching 
adhesive), each Group being subdivided into two subgroups according to the restoration method, A: composite resin; B: Flowable resin base, composite resin restoration and surface sealer. All trial bodies were sealed at the apex and on the entire external surface with two layers of nail polish up to $1 \mathrm{~mm}$ from the tooth-restoration interface, thermocycled between $5{ }^{\circ} \mathrm{C}$ and $55^{\circ} \mathrm{C}$, immersed in methylene blue at $2 \%$ for 24 hours and cut transversely in the palatal / lingual vestibule direction to measure in millimeters. Results: The data were statistically analyzed with IBM SPSS stadicts version 2.0, applying double classification ANOVA; a statistically significant difference $(\mathrm{p}<0.05)$ was observed between the subgroups according to the restoration method. Conclusions: the use of base and surface sealer in combination with the composite resin restoration decreased microfiltration by applying both the adhesive system with previous acid etching and the self-etched adhesive system, while the highest microleakage was obtained with composite resin (without base or sealer surface) and the use of the self-etching adhesive system.

KEYWORDS: microfiltration, composite resins, adhesives.

\section{INTRODUCCIÓN}

La incorporación de la técnica adhesiva en la odontología restauradora permitió el desarrollo de múltiples técnicas y materiales, que apuntan actualmente a la preservación máxima de la estructura dental, equilibrando función, estética y salud bucal (1). Sin embargo, a pesar de las mejoras en las propiedades de las resinas compuestas, la contracción de polimerización de dichos compuestos crea tensiones alrededor de la interfase, lo que finalmente generamicrofiltraciones (2), permitiendo el ingreso de las bacterias y diversas complicaciones posoperatorias, tales como afecciones pulpares debido a la formación de caries secundaria en la interfase diente-restauración, destrucción del tejido dentario con cada nuevo tratamiento y fracaso del material restaurador (3). La disminución de la contracción de la polimerización pueden obtenerse mediante una técnica de estratificación oblicua con incrementos o diseños de cavidades con un factor $\mathrm{C}$ bajo (4), mientras que el uso de la técnica incremental o estratificada apropiada puede reducir el desarrollo de caries secundarias, dando como resultado un mejor sellado marginal, y por ende disminuir la microfiltración (5). Algunos estudios reportan la efectividad de resinas compuestas fluidas como base o revestimiento en la reducción de microfiltraciones, debido a su fluidez y módulo de elasticidad bajo que permite absorber el "stress" de la contracción de polimerización de las resinas compuestas asegurando la continuidad en la superficie adhesiva y la posterior reducción de las microfiltraciones $(6,7)$, refiriendo incluso en otras investigaciones, que la variación del grosor de dicho revestimiento también influye, Hernándes et al., reportaron que el espesor inferior $(1 \mathrm{~mm})$ del compuesto fluido proporcionó menos microfiltración $(\mathrm{p} \leq 0,05)(8)$. Teniendo en cuenta que el acabado y pulido también pueden originar microgrietas en la superficie de la restauración con resina, generando a su vez microfiltraciones, Hepdeniz, Temel, Ugurlu, y Koskanevaluaron la capacidad de sellado marginal utilizando diferentes selladores resinosos aplicados en la superficie de las restauraciones de resina compuesta, reportaron que los selladores superficiales mostraron significativamente menos microfiltración $(p<0,05)(9)$. Otro factor que también pude contribuir a la presencia de microfiltración son los sistemas adhesivos, con los adhesivos de 5ta generación la adhesión se logra a través del grabado ácido previo, mientras que las siguientes generaciones se desarrollaron con el fin de superar los problemas asociados con el uso del grabado y enjuague, la sensibilidad postoperatoria y simplificando el accionar clínico con una manipulación másrápida, pero con una disminución de la acidez lo que podría llegar a comprometer el éxito de la adhesiónen esmalte $(10,11)$, varias investigaciones coinciden en que las restauraciones son clínicamente aceptables cuando se usan resinas híbridas, nanoparticulas o microhíbridas y sistemas adhesivos convencionales (12). La relevancia de este estudio se basa en la evaluación de la microfiltración de restauraciones con resina realizadas con cuatro técnicas que difieren en cuanto a protocolo y materiales utilizados, se pretende seleccionar, recomendar y transferir a la clínica la más adecuada con el fin de obtener menor microfiltración evitando las complicaciones que ella conlleva. El objetivo de esta investigación fue comparar la profundidad de microfiltraciones en restauraciones con resina Clase I variando el método de restauración y el sistema adhesivo utilizado. 


\section{MATERIAL Y MÉTODOS}

Se realizó un estudio experimental ex vivo, controlado aleatorizado, previa aprobación del Comité de Ética de de la Facultad de Odontología "Pierre Fauchard" de la Universidad Autónoma del Paraguay. Se utilizaron 128 molares humanos superiores e inferiores con la corona íntegra, sin lesiones cariosas, sin restauraciones previas, ni alteraciones estructurales o morfológicas en la cara oclusal, seleccionados aleatoriamente del Banco de dientes de la Facultad de Odontología de la Universidad Autónoma "Pierre Fauchard". Fueron almacenados en un recipiente cerrado con agua destilada a temperatura ambiente que fue cambiada cada 3 días para su conservación e hidratación hasta el momento de la experimentación. Se realizó la limpieza y desinfección de cada órgano dentario y el almacenamiento en hipoclorito de sodio al 2,5\%, luego del retiro de los elemento orgánicos e inorgánicos de forma manual con curetas, lavado con jabones neutros con ayuda de cepillitos profilácticos y ultrasonido, y esterilización con autoclave a $115^{\circ} \mathrm{C}$ por 40 minutos, en cada pieza dentaria se realizó una preparación de Clase I con las siguientes dimensiones: $4 \mathrm{~mm}$ en sentido mesiodital, $4 \mathrm{~mm}$ en sentido ocluso palatino/ lingual y $3 \mathrm{~mm}$ de profundidad mediante el uso de fresa redonda diamantada, constatando las medidas con sonda periodontal y una regla milimetrada. Los cuerpos de prueba fueron distribuidos en forma aleatoria en grupos según el sistema adhesivo aplicado y el método de restauración:

Grupo I (control): grabado ácido (Scotchbond ${ }^{\mathrm{TM}}$ $37 \%$ ) previo a la aplicación del sistema adhesivo (Adper ${ }^{\mathrm{TM}}$ Single Bond 2).

Grupo II: aplicación del sistema adhesivo autograbado (Single Bond® Universal).

Posterior a la aplicación de los agentes de unión, cada grupo se dividió en dos subgrupos:

Subgrupo A: restauración en incrementos oblicuos con resina compuesta (Filtek ${ }^{\mathrm{TM}} \mathrm{P} 60$ ) y pulido con gomas de grano grueso y fino.

Subgrupo B: $1 \mathrm{~mm}$ de resina fluida en el piso de la preparación (Filtek ${ }^{\mathrm{TM}}$ Z350 XT FLOW), restauración en incrementos con resina compuesta (Filtek ${ }^{\mathrm{TM}}$ P60), pulido, y posterior sellado superficial también con resina fluida (Filtek ${ }^{\mathrm{TM}}$ Z350 XT FLOW).

Los cuerpos de prueba fueron sometidos a un proceso de termociclado de 500 ciclos, en baños de agua entre $5{ }^{\circ} \mathrm{C}$ y $55^{\circ} \mathrm{C}$ durante 30 segundos y temperamentándose a $24{ }^{\circ} \mathrm{C}$ durante 15 segundos siguiendo las normas ISO/TS 11405:2015. Luego del termociclado fueron sumergidas en azul de metileno al $2 \%$ durante 24 horas a temperatura ambiente, lavadas con abundante agua y finalmente cortados transversalmente con un disco de diamante a baja velocidad en sentido vestíbulo/palatino o lingual, obteniéndose dos fragmentos por cada muestra y cuatro interfases a medir. Cada fragmento fue fotografiado con una cámara réflex Cannon con un protocolo estandarizado para todas la muestras y con el programa informático ImagenJ 1.50i año 2016, se realizaron las mediciones traduciendo los pixeles a distancias, previa calibración de cada imagen. Con las herramientas disponibles en el programa informático se definió una dimensión ya conocida de $3 \mathrm{~mm}$, luego se procedió a medir la distancia en milímetros del ingreso del azul de metileno en cada interfase dienterestauración. Los datos recolectados fueron analizados estadísticamente con el programa informático IBM SPSS stadicts versión 2.0. utilizando como prueba estadística el ANOVA de doble clasificación y la prueba de Tukey B y Duncan, previa realización de la prueba de normalidad de Kolmogorov-Sminov y de homogeneidad de Levene. Las hipótesis nula considerada fue "no hay diferencias en la profundidad de microfiltración entre las diferentes técnicas de restauración" y la alternativa "existen diferencias en la profundidad de microfiltración entre las diferentes técnicas de restauración".

\section{RESULTADOS}

Las mediciones de las microfiltraciones de los grupos se muestran en el gráfico 1, donde se observa que la utilización de resina fluida y sellador superficial disminuyeron la microfiltración, usando tanto el sistema adhesivo con grabado ácido como el autograbado $(1,87501 \pm 0,0744 \mathrm{~mm}$ y $1,82813 \pm$ $0,07708 \mathrm{~mm}$ respectivamente). Para la estadística diferencial se aplicaron en una primera etapa las pruebas de normalidad de Kolmogorov-Smirnov y la de homogeneidad de Levene, aceptándose la hipótesis de normalidad en ambos casos ( $p=0,574$ y 


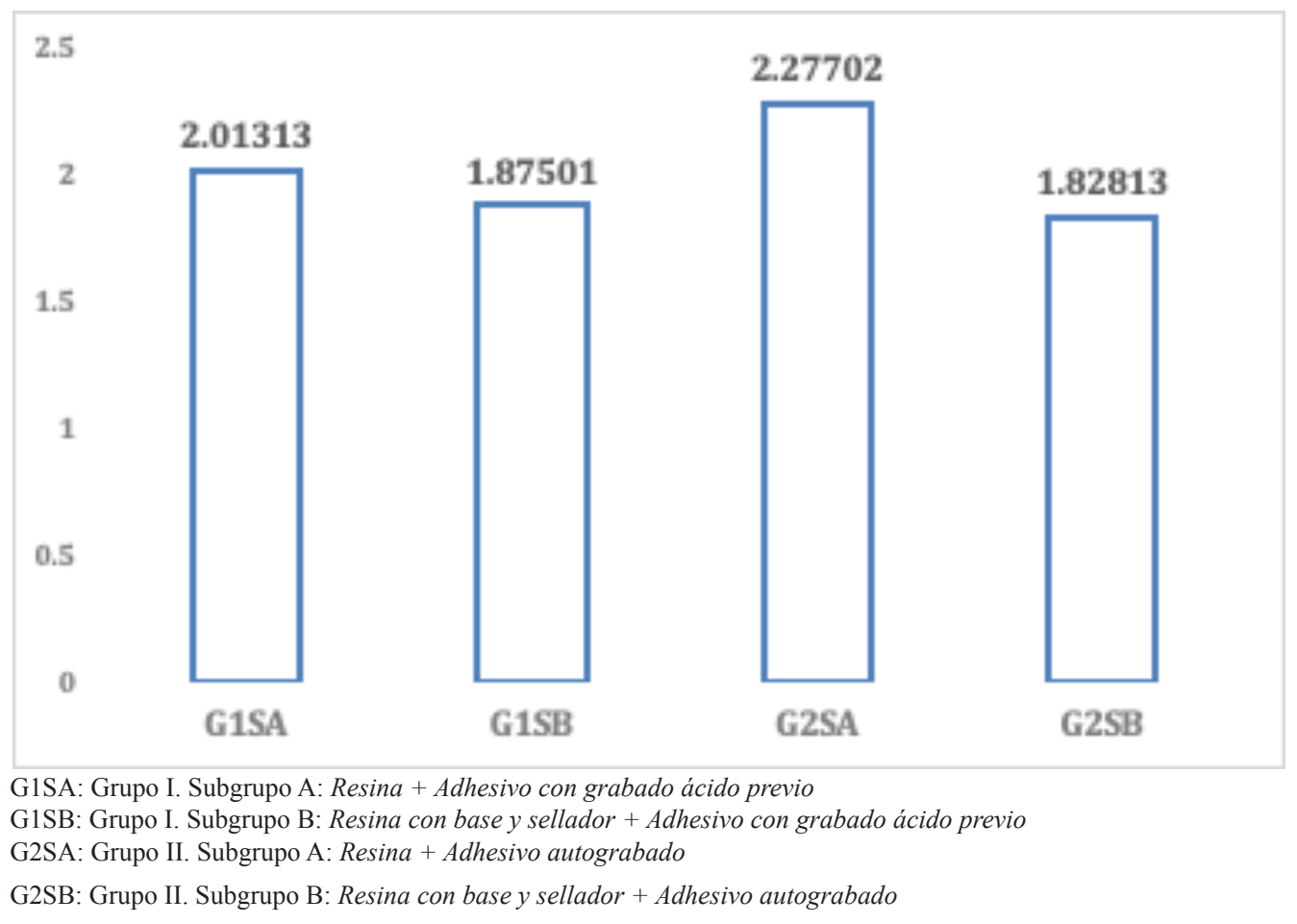

Gráfico 1. Distribución de la media de microfiltración en milímetros $(\mathrm{mm})$ según el método de restauración y el método de aplicación del adhesivo.

$\mathrm{p}=0,181$ ), lo que permitió la aplicación del ANOVA de doble clasificación con dos factores independientes (sistema adhesivo y método de restauración), donde se constató diferencias estadísticamente significativas entre los subgrupos de este estudio con un valor de $p<0,05$. Para diferenciar que subgrupos difieren significativamente se utilizó la prueba de Tukey B y Duncan, donde la mayor microfiltración se obtuvo con resina compuesta ( $\sin$ base ni sellador superficial) y con la utilización del sistema adhesivo autograbado (sin grabado ácido previo) con una media de 2,27702 $\mathrm{mm} \pm 0,08481 \mathrm{~mm}$.

\section{DISCUSIÓN}

Los resultados obtenidos en este estudio tienen semejanza con Falconi et al. (11), quienes reportaron la existencia de diferencias de microfiltración entre los grupos evaluados ( $p>0,05)$, de forma más evidente a nivel cervical, independientemente del sistema adhesivo empleado al evaluar restauraciones con resina compuesta en cavidades Clase V, utilizando dos sistemas adhesivos de diferente composición, en el primer grupo aplicó acondicionamiento ácido y luego el sistema adhesivo, mientras que en el segundo grupo se aplicó un adhesivo autograbado.

Por otro lado, Licla yAlbites compararon el grado de microfiltración de un sellante resinoso usando diferentes sistemas adhesivos donde obtuvieron el porcentaje de mayor grado de microfiltración con los adhesivos de séptima generación o autograbado $(80 \%)$ y el de menor microfiltración con los adhesivos de quinta generación o con grabado ácido previo (20\%) (12), contrastando con los resultados de este estudio, donde incluso el autograbado presento valores levemente menores de microfiltración, pero al ser combinados con otras técnicas de restauración (sellador superficial y utilización de bases) lo que pudo haber favorecido la efectividad del sistema adhesivo autograbado.

También hay semejanza con Scotti et al., (13), que evaluaron la capacidad de sellado marginal de un compuesto de resina fluida en sustratos de esmalte y dentina, utilizando el sistema adhesivo con grabado ácido previo y de dos componentes (primer y adhesivo por separado), en los resultados reportaron que las 
resinas fluidas proporcionaron un mejor sellado marginal en la dentina, siendo estadísticamente significativas para el sustrato $(\mathrm{p}=0,0001)$, aunque mostraron valores de microfiltración similares a los márgenes del esmalte. Es importante considerar que las características del sustrato podrían influir en el sellado marginal, ya que el esmalte posee una superficie adhesiva mayor, debido al alto contenido inorgánico, en comparación a la dentina con mayor cantidad de colágeno y agua, que comprometen la adhesión.

Existe coincidencia con Lokhande et al., estudio en que se analizaron la efectividad de las resinas fluidas como base en la reducción de las microfiltraciones, donde reportaron menor microfiltración con el uso de un revestimiento fluido bajo la resina hibrida y compactable en comparación con el grupo sin revestimiento (14) y con Hernández et al., que al evaluar cavidades de Clase II restauradas con resina compuesta mediante la variación de grosor de las resinas fluidas utilizadas como base, concluyeron que el uso de un grosor inferior $(1 \mathrm{~mm})$ de una resina compuesta fluida como revestimiento proporcionó menos microfiltración (8). Las resinas fluidas contienen partículas de relleno más pequeñas con reducción del volumen para lograr dicha fluidez, lo que a su vez aumenta la contracción de polimerización, razón por la cual el espesor mínimo podría controlar mejor este efecto.

Si existe diferencias con Poggio et al. (15) que evaluaron la microfiltración en restauraciones Clase II utilizando diferentes resinas fluidas (FiltekFlowable, GrandioSOFlow, SDR Flow y SonicFill) como base y restaurándolas con sus respectivas resinas compuestas y el sistema adhesivo Adper Scotchbond XT con grabado ácido previo, comparándolos con un grupo control sin revestimiento; en los resultados reportaron que no existían diferencias significativas entre el grupo control sin revestimiento, esto podría deberse a utilización de grabado ácido en todos los grupos, y de las resinas compuestas nanohíbridas que influyeron en la reducción de la microfiltración debido a la disminución de partículas de las mismas, lo que reduce la contracción de polimerización y por ende la microfiltración.

Los resultados obtenidos difieren con Arlan et al., que evaluaron el efecto de la aplicación de la resina fluida como revestimiento y variando el tipo de sistema adhesivo aplicado (autograbado de dos pasos, autograbado de un paso y con grabado ácido total previo) en Clase $\mathrm{V}$, y restauradas con una resina compuesta híbrida, donde no se encontraron diferencias estadísticamente significativas entre los grupos ( $p>0,05)(16)$; pero si se asemejan a Reddy, Jayashankar, Nainan y Shivanna (17) que compararon la restauración de composites Clase II utilizando resinas fluidas con y sin revestimiento, pero utilizando el sistema adhesivo con grabado ácido previo a la aplicación de dos capas de Single Bond y restauradas con resina compuesta microhíbrida condensable P60; concluyeron que los especímenes con revestimiento de composite fluido de 0,5 a $1 \mathrm{~mm}$ mostraron menos microfiltración en comparación al grupo control ( $\sin$ revestimiento). Por lo tanto, también se puede considerar que las resinas microhíbridas son las que tienen menos contracción de polimerización (por su alto porcentaje de relleno), debido a la disminución del tamaño de sus partículas y mejoras en sus propiedades, lo que influye finalmente en el grado de microfiltración.

\section{CONCLUSIONES}

En este estudio la utilización de base y sellador superficial en combinación con la restauración de resina compuesta disminuyeron la microfiltración, tanto con la aplicación del sistema adhesivo con grabado ácido previo, como con la aplicación del sistema adhesivo autograbado. Las diferencias observadas en la profundidad de microfiltración entre las diferentes técnicas de restauración fueron estadísticamente significativas $(p<0,05)$. Dentro de las limitaciones del estudio se menciona la medición realizada en un solo corte longitudinal, por lo que podría haber alguna variación en el avance de la microfiltración considerando toda la extensión de la interfase diente-restauración.

Conflicto de intereses: los autores no tienen conflicto de interés con este informe.

Aprobación de ética: Ausente

Financiamiento: Ninguno.

Contribuciones de los autores: todos los autores contribuyeron a este manuscrito. 


\section{Correspondencia}

Laura María Cáceres Díaz

Dirección: Willams Richardson $N^{\circ} 229$ c/ Sajonia.

Asunción-Paraguay

Teléfono: 595983447817

Correo electrónico: laurymcd45@gmail.com

\section{REFERENCIAS BIBLIOGRAFICAS}

1. Chaple-Gil AM, Gispert-Abreu EA. Recomendaciones para el empleo práctico de resinas compuestas en restauraciones estéticas. Rev Cubana Estomatol. 2015; 52(3): 293-313.

2. Sarfi S, Sharma N, Carg E, Bali D. Comparing microleakage in silorane based composite and nanofilled composite using different layering techniques in class I restorations: An in vitro study. IAIM. 2017; 4(7): 23-32.

3. Sarfi S, Bali D, Grewal MS. Effect of different layering techniques on microleakage of nanofilled composite in class I restorations: An In vitro study. J Int Clin Dent Res Organ. 2017; 9(1): 8-11.

4. Swampa MU, Koshy S, KumarA, Nanjappa N, Benjamin $\mathrm{S}$, Nainan MT. Comparing marginal microleakage of three BulkFill composites in Class II cavities using confocal microscope: An in vitro study. J Conserv Dent. 2015; 18(5): 409-413.

5. Katona A, Barrak I. Comparison of composite restoration techniques. Interdisciplinary Description of Complex Systems. 2016; 14(1): 101-115.

6. Chaple-Gil AM. Técnica modificada de restauración de cavidades clase II utilizando resinas compuestas.

Revista Habanera de Ciencias Medicas. 2015; 14(3): 337-347.

7. Aggarwal V, Singla M, Yadav S, Yadav H. Effect of flowable composite liner and glass ionomer liner of class II gingival adaptation of direct composite restoratios with different bondig strategies. Journal of Dentistry. 2014; 4(2): 619-625.

8. Hernándes N, Catelan A, Soares G, et al. Influence of flowable composite and restorative technique on microleakage of class II restorations. JICD. 2014; 5(4): 283-288.
9. Hepdeniz OK, Temel UB, Ugurlu M, Koskan O. The effect of surface sealants with different filler contento $\mathrm{n}$ microleakage of Class $\mathrm{V}$ resin composite restorations. European Journal of Dentistry. 2016; 10(2): 163-169.

10. Amoedo-Campos-Velo MM, Braga-Ferraz-Coelho LV, Tarkany-Basting R, Botelho-Do-Amaral FL, Gomes-Franca FM. Longevity of restorations in direct composite resin: literatura review. RevistaGaúcha de Otontología. 2016; 64(3): 320-326.

11. Falconi-Borja GM, Molina-Pule CG, VelásquezRon BV, Armas-Vega AC. Evaluación del grado de microfiltración en restauraciones de resina compuesta comparando dos sistemas adhesivos tras diferentes periodos de envejecimiento. Rev Fac Odontol Univ Antioq. 2016; 27 (2): 281-295

12. Licla K, Albites U. Grado de filtración de un sellante resinoso con diferentes sistemas adhesivos. OdontolPediatr. 2015; 14(2): 120-128.

13. Scotti N, Comba A, Gambino A, et al. Microleakage at enamel and dentin margins with a bulk fills flowable resin. European Journal of Dentistry. 2014; 8(1): 1-8.

14. Lokhande NA, Padmai AS, Rathore VPS, Shingane S, Jayashankar DN, Sharma U. Effectiveness of flowable resin composite in reducing microleakage - An in vitro study. Journal of International Oral Healh. 2014; 6(3): 111-114.

15. Poggio C, Chiesa M, Scribante A, Mekler J, Colombo M. Microleakage in Class II composite restorations with margins below the CEJ: In vitro evaluation of different restorative techniques. Med Oral Patol Oral Cir Bucal. 2013; 18(5): 793-798.

16. Arlan S, Demirbuga S, Ustun Y, Dincer AN, Canakci $\mathrm{BC}$, Zorba YO. The effect of a new generation of resin composed of fluid in microleakage in class $\mathrm{V}$ composite restorations as an intemediate layer. J Conserv Dent. 2013; 16(3): 189-193.

17. Reddy SN, Jayashandar DN, Nainan M, Shivanna V. The effect of flowable composite lining thickness with various curing tecniques on microleakage in class II composite restorations: An in vitro study. J Contemp Dent Pract. 2013; 14(1): 56-60.

Recibido : 15-08-2021

Aceptado : 27-09-2021 